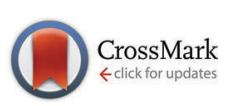

Cite this: Phys. Chem. Chem. Phys., $2016,18,8243$

DOI: $10.1039 / \mathrm{c} 6 \mathrm{cp} 90057 \mathrm{~g}$

www.rsc.org/pccp

\section{Correction: Spectroscopic characteristics of the OSIRIS near-backscattering crystal analyser spectrometer on the ISIS pulsed neutron source}

\author{
Mark T. F. Telling, ${ }^{\text {a }}$ Stuart I. Campbell, $\uparrow^{a}$ Dennis Engberg, ${ }^{a}$ \\ David Martín y Marero ${ }^{\text {bcd }}$ and Ken $\mathrm{H}$. Andersen $\ddagger^{\mathrm{e}}$ \\ Correction for 'Spectroscopic characteristics of the OSIRIS near-backscattering crystal analyser \\ spectrometer on the ISIS pulsed neutron source' by Mark T. F. Telling et al., Phys. Chem. Chem. Phys., \\ $2005,7,1255-1261$
}

The authors are pleased to include Stuart I. Campbell, Dennis Engberg and David Martín y Marero on the manuscript. The corrected list of authors and affiliations for this paper is as shown above.

The Royal Society of Chemistry apologises for these errors and any consequent inconvenience to authors and readers.

\footnotetext{
${ }^{a}$ ISIS Facility, Rutherford Appleton Laboratory, Chilton, Oxon, UK OX11 OQX. E-mail: mark.telling@stfc.ac.uk

${ }^{b}$ Departamento de Física Aplicada, Facultad de Ciencias, Universidad Autónoma de Madrid, 28049 Madrid, Spain

${ }^{c}$ Fundación Parque Científico de Madrid, Campus de Cantoblanco, 28049 Madrid, Spain

${ }^{d}$ Centro de Microanálisis de Materiales \& Instituto Nicolás Cabrera, Universidad Autónoma de Madrid, 28049 Madrid, Spain

${ }^{e}$ Institut Laue-Langevin, Grenoble, France

$\dagger$ Current address: Neutron Data Analysis \& Visualization Division, Oak Ridge National Laboratory, Oak Ridge, TN, USA.

\# Current address: European Spallation Source ERIC, P.O. Box 176, 22100 Lund, Sweden.
} 